\title{
Towards numerical relativity in scalar Gauss-Bonnet gravity: $3+1$ decomposition beyond the small-coupling limit
}

\author{
Helvi Witek $\odot,{ }^{1,2, *}$ Leonardo Gualtieri $\oplus^{3, \dagger}$ and Paolo Pani $\oplus^{3, \$}$ \\ ${ }^{1}$ Department of Physics, University of Illinois at Urbana-Champaign, Urbana, Illinois 61801, USA \\ ${ }^{2}$ Department of Physics, King's College London, Strand, London WC2R 2LS, United Kingdom \\ ${ }^{3}$ Dipartimento di Fisica, "Sapienza" Universitá di Roma \& Sezione INFN Roma 1, \\ P.A. Moro 5, 00185 Roma, Italy
}

(Received 13 April 2020; accepted 29 May 2020; published 25 June 2020)

\begin{abstract}
Scalar Gauss-Bonnet gravity is the only theory with quadratic curvature corrections to general relativity whose field equations are of second differential order. This theory allows for nonperturbative dynamical corrections and is therefore one of the most compelling case studies for beyond-general relativity effects in the strong-curvature regime. However, having second-order field equations is not a guarantee for a healthy time evolution in generic configurations. As a first step toward evolving black-hole binaries in this theory, we here derive the $3+1$ decomposition of the field equations for any (not necessarily small) coupling constant, and we discuss potential challenges of its implementation.
\end{abstract}

DOI: 10.1103/PhysRevD.101.124055

\section{INTRODUCTION}

Gravitational-wave (GW) observations are providing us with novel tests of general relativity (GR) in the strongfield/highly dynamical regime and of fundamental physics at large [1-5]. These tests will become increasingly more accurate in the near future, owing to a better sensitivity of the GW interferometers and to the large number of mergers to be detected in future runs.

While there is no shortage of observational data, the theoretical modeling of beyond-GR effects in the nonlinear regime of gravity is the real bottleneck of this kind of tests. Indeed, current tests of gravity based on the inspiralmerger-ringdown signal from a black-hole coalescence either adopt phenomenological waveforms [6,7] or focus separately on the phases of the coalescence that can be studied perturbatively, namely the inspiral [8] and the ringdown [9] (see Refs. [2,5] for further reviews). This is due to the fact that studying a black-hole coalescence in a modified theory of gravity is a formidable task that has only recently been attacked for a few theories admitting a perturbative treatment of the field equations [10-13]. These studies will be highly informative to develop a consistent inspiral-merger-ringdown waveform in extensions of GR at the perturbative level, but fail to capture any possible nonperturbative dynamics that might significantly affect the GW signal precisely in the hitherto poorly explored merger phase. An example of such a nonperturbative effect is the

\footnotetext{
*hwitek@illinois.edu

†leonardo.gualtieri@roma1.infn.it

paolo.pani@uniroma1.it
}

dynamical scalarization in neutron-star binaries in some scalar-tensor theories [14-16], and a similar effect is expected for binary black holes in a certain class of theories with quadratic curvature corrections $[17,18]$.

It is thus of utmost importance to study extensions of GR in their full glory, i.e., beyond a perturbative regime. However, in such an attempt one would face two major challenges. First, many extensions of GR are constructed as effective field theories and, as such, they are perturbative by construction [2]; if treated nonperturbatively, these theories lead to instabilities and other pathologies [19]. Second, even for the subclass of theories that are not manifestly pathological, it is unclear (i) how to set up an initial-value problem, i.e., how to write the field equations as a set of first-order-in-time independent differential equations (in this case we call the problem "well formulated"); (ii) if the problem is well posed, i.e., if it admits a unique solution with continuous dependence on given initial data [20]. Proving well-posedness of a theory is very challenging (see, e.g., [21]) and, in fact, solving this problem in GR took several decades (see Ref. [22-24] for a review). A proof of well-posedness beyond GR has been recently obtained, but only for the simplest theories (namely, the socalled Bergmann-Wagoner scalar-tensor theories [25,26]), in Einstein-Æther theory [27], and for higher derivative theories such as Horndeski or Lovelock gravity in the weak-field regime [28-30]. Clearly, extending such results to a well-motivated, nonperturbative modified theory of gravity would be extremely important.

With these motivations in mind, here we study scalar Gauss-Bonnet (sGB) gravity-the only theory with quadratic curvature corrections to GR whose field equations are 
of second differential order [4,31-33]. While this theory can be studied perturbatively $[34,35]$, its differential structure does not make it manifestly pathological even when treated exactly. However, having second-order field equations is not a guarantee for a healthy time evolution in generic configurations [36,37]. This has only been shown in spherically symmetric configurations [38,39], where it was found that the character of the equations governing the spherical collapse in sGB changes from hyperbolic to elliptic in some spacetime regions and for open sets of initial data.

As a first step toward evolving black-hole binaries, here we present the $3+1$ decomposition of the field equations for any (not necessarily small) coupling constant, and we preliminarily discuss the possibility of a well-formulated and well-posed time evolution.

\section{ACTION AND EQUATIONS OF MOTION}

The action describing sGB gravity involving a real, massless scalar field $\Phi$ is given by [4,31-33]

$S=\frac{1}{16 \pi} \int \mathrm{d}^{4} x \sqrt{-g}\left[{ }^{(4)} R-\frac{1}{2}(\nabla \Phi)^{2}+\frac{\alpha_{\mathrm{GB}}}{4} f(\Phi) \mathcal{R}_{\mathrm{GB}}\right]$,

where ${ }^{(4)} R$ is the four-dimensional Ricci scalar, $\alpha_{\mathrm{GB}}$ is the dimensionful coupling constant, and $f(\Phi)$ is a function coupling the scalar field to the Gauss-Bonnet (GB) invariant

$$
\mathcal{R}_{\mathrm{GB}}={ }^{(4)} R^{2}-4^{(4)} R_{a b}{ }^{(4)} R^{a b}+{ }^{(4)} R_{a b c d}{ }^{(4)} R^{a b c d} .
$$

${ }^{(4)} R_{a b c d}$ and ${ }^{(4)} R_{a b}$ are the four-dimensional Riemann and Ricci tensors, respectively. In the following we will employ geometric units $G=1=c$. Typical choices of the scalar function are (i) the dilaton coupling $f(\Phi)=e^{\Phi}[32]$ (which also appears in low-energy effective actions from string theory); (ii) the linear coupling $f(\Phi)=\Phi$, for which the theory is shift symmetric [4,40], i.e., invariant for $\Phi \rightarrow \Phi+$ const; and (iii) the class of couplings for which $f^{\prime}(0)=0$, such as $f(\Phi)=\Phi^{2}$ and $f(\Phi)=e^{\Phi^{2}}-1$ $[17,18,41,42]$, which can lead to spontaneous scalarization of black holes, i.e., to dynamical formation of nonperturbative scalar field configurations.For other possible choices of the coupling function, see, e.g., Refs. [43,44]. In this paper we shall consider general coupling functions. We shall not consider more complicated sGB gravity theories, such as those with a scalar potential $[45,46]$ or those with further coupling terms linear in the curvature tensor [47].

In the limit $\alpha_{\mathrm{GB}} \rightarrow 0$, sGB gravity reduces to GR with a minimally coupled scalar field; the modification of GR is thus given by the GB coupling term $\alpha_{\mathrm{GB}} f(\Phi) \mathcal{R}_{\mathrm{GB}}$. While the theory can be studied in a perturbative regime where $\alpha_{\mathrm{GB}} f(\Phi) \mathcal{R}_{\mathrm{GB}} \ll{ }^{(4)} R$, here we do not assume this small-coupling limit and are interested in the case in which the constant $\alpha_{\mathrm{GB}}$ can take any finite value. For instance, in the case of a stationary black hole of mass $M$, the dimensionless quantity $\alpha_{\mathrm{GB}} / M^{2}$ can be as large as $\sim 0.1-1[32,33,40,48]$.

Varying the action (1) with respect to the scalar field $\Phi$ and metric $g^{a b}$ yields the field equations

$$
\begin{aligned}
& \square \Phi=-\frac{\alpha_{\mathrm{GB}}}{4} f^{\prime}(\Phi) \mathcal{R}_{\mathrm{GB}}, \\
& G_{a b}=\frac{1}{2} T_{a b}^{\Phi}-\frac{\alpha_{\mathrm{GB}}}{8} \mathcal{G}_{a b},
\end{aligned}
$$

where $f^{\prime} \equiv \mathrm{d} f / \mathrm{d} \Phi, G_{a b}={ }^{(4)} R_{a b}-1 / 2 g_{a b}{ }^{(4)} R$, and the canonical scalar field energy-momentum tensor is

$$
T_{a b}^{\Phi}=\nabla_{a} \Phi \nabla_{b} \Phi-\frac{1}{2} g_{a b} \nabla^{c} \Phi \nabla_{c} \Phi
$$

The modification due to the GB term reads $[32,33]$

$$
\begin{aligned}
\mathcal{G}_{a b}= & 2 g_{c(a} g_{b) d} \epsilon^{e d f g} \nabla_{h}\left[{ }^{*} R^{c h}{ }_{f g} f^{\prime} \nabla_{e} \Phi\right] \\
= & 16^{(4)} R^{c}{ }_{(a} \mathcal{C}_{b) c}+8 \mathcal{C}^{c d}\left({ }^{(4)} R_{a c b d}-g_{a b}^{(4)} R_{c d}\right) \\
& -8 \mathcal{C} G_{a b}-4{ }^{(4)} R \mathcal{C}_{a b},
\end{aligned}
$$

where ${ }^{*} R^{a b}{ }_{c d}=\epsilon^{a b e f(4)} R_{e f c d}$ is the dual Riemann tensor, $\epsilon^{a b c d}$ is the totally antisymmetric Levi-Civita symbol, and we have defined the tensor

$$
\mathcal{C}_{a b}=\nabla_{a} \nabla_{b} f(\Phi)=f^{\prime} \nabla_{a} \nabla_{b} \Phi+f^{\prime \prime} \nabla_{a} \Phi \nabla_{b} \Phi,
$$

with $\mathcal{C}=g^{a b} \mathcal{C}_{a b}$. To derive the time evolution formulation of sGB gravity we employ the gravitoelectric and gravitomagnetic decomposition of the four-dimensional Weyl tensor $W_{a b c d}$. In terms of the latter, the GB invariant $\mathcal{R}_{\mathrm{GB}}$ and the tensor $\mathcal{G}_{a b}$ can be expressed as

$$
\begin{aligned}
\mathcal{R}_{\mathrm{GB}}= & W_{a b c d} W^{a b c d}-2^{(4)} R_{a b}{ }^{(4)} R^{a b}+\frac{2}{3}{ }^{(4)} R^{2} \\
\mathcal{G}_{a b}= & 8^{(4)} R^{c}{ }_{(a} \mathcal{C}_{b) c}-4 \mathcal{C}^{(4)} R_{a b}-\frac{8}{3}{ }^{(4)} R\left(\mathcal{C}_{a b}-g_{a b} \mathcal{C}\right) \\
& +8 \mathcal{C}^{c d}\left(W_{a c b d}-\frac{1}{2} g_{a b}{ }^{(4)} R_{c d}\right)
\end{aligned}
$$

\section{TIME EVOLUTION FORMULATION}

We here derive a formulation of the sGB field equations (3) as a time evolution problem. We therefore extend standard methods of numerical GR in $3+1$ dimensions; see, e.g., [49]. 


\section{A. Decomposition of the spacetime}

The basis of any formulation of a gravitational theory as a time evolution problem is the decomposition of spacetime into a set of spatial hypersurfaces $\left(\Sigma_{t}, \gamma_{i j}\right)$ labeled by a time parameter $t$ and with 3-metric $\gamma_{i j}$ given by the space components of $\gamma_{a b}=g_{a b}+n_{a} n_{b}$. Here $n^{a}$ denotes the timelike unit vector normal to the hypersurface and is normalized to $n^{a} n_{a}=-1$. The spatial metric defines a projection operator

$$
\gamma_{a}^{b}=\delta_{a}^{b}+n^{a} n_{b},
$$

with $\gamma_{a}^{b} n^{b}=0$ by construction. The line element takes the form

$$
\begin{aligned}
\mathrm{d} s^{2} & =g_{a b} \mathrm{~d} x^{a} \mathrm{~d} x^{b} \\
& =-\left(\alpha^{2}-\beta^{k} \beta_{k}\right) \mathrm{d} t^{2}+2 \gamma_{i j} \beta^{i} \mathrm{~d} t \mathrm{~d} x^{j}+\gamma_{i j} \mathrm{~d} x^{i} \mathrm{~d} x^{j},
\end{aligned}
$$

where $\alpha$ and $\beta^{i}$ are the lapse function and shift vector, respectively. We denote the covariant derivative and Riemann curvature tensor associated with the spatial metric $\gamma_{i j}$ by $D_{i}$ and $R_{i j k l}$, respectively. Similarly, $R_{i j}$ and $R$ are, respectively, the Ricci tensor and the Ricci scalar associated with the spatial metric. To complement the description of spacetime we introduce the extrinsic curvature

$$
K_{i j}=-\gamma^{c}{ }_{i} \gamma^{d}{ }_{j} \nabla_{c} n_{d}=-\frac{1}{2} \mathcal{L}_{n} \gamma_{i j},
$$

where $\mathcal{L}_{n}=\frac{1}{\alpha}\left(\partial_{t}-\mathcal{L}_{\beta}\right)$ is the Lie derivative along $n^{a}$ and $\mathcal{L}_{\beta}$ the Lie derivative along the shift vector $\beta^{i}$. Analogously, we introduce the "momentum" associated with the scalar field

$$
K_{\Phi}=-\mathcal{L}_{n} \Phi .
$$

We now proceed by deriving the equations of sGB gravity in terms of $\left(\gamma_{i j}, \Phi\right)$ and their momenta $\left(K_{i j}, K_{\Phi}\right)$, combined with an appropriate gauge choice for $\left(\alpha, \beta^{i}\right)$.

\section{B. Dynamical variables}

In this section we summarize a set of new dynamical variables and shorthand notations that we will use in the derivation of the sGB time evolution problem.

Decomposition of the auxiliary scalar field tensor: We decompose the auxiliary tensor $\mathcal{C}_{a b}$ given in Eq. (6) into its normal and spatial components. The different projections with the operator defined in Eq. (8) yield

$$
\begin{aligned}
C_{\mathrm{nn}} & =\mathcal{C}_{a b} n^{a} n^{b} \\
& =f^{\prime \prime} K_{\Phi}^{2}-\frac{f^{\prime}}{\alpha} D^{k} \alpha D_{k} \Phi-f^{\prime} \mathcal{L}_{n} K_{\Phi}, \\
C_{i} & =-\gamma^{a}{ }_{i} n^{b} \mathcal{C}_{a b} \\
& =f^{\prime \prime} K_{\Phi} D_{i} \Phi+f^{\prime} D_{i} K_{\Phi}-f^{\prime} K^{j}{ }_{i} D_{j} \Phi,
\end{aligned}
$$

$$
\begin{aligned}
C_{i j} & =\gamma^{a}{ }_{i} \gamma^{b}{ }_{j} \mathcal{C}_{a b} \\
& =f^{\prime}\left(D_{i} D_{j} \Phi-K_{\Phi} K_{i j}\right)+f^{\prime \prime} D_{i} \Phi D_{j} \Phi .
\end{aligned}
$$

Trace-free decomposition: We further decompose the extrinsic curvature $K_{i j}$ and the intrinsic (spatial) curvature determined by the three-dimensional Ricci tensor $R_{i j}$ into their trace and trace-free parts

$$
K_{i j}=A_{i j}+\frac{1}{3} \gamma_{i j} K, \quad R_{i j}=R_{i j}^{\mathrm{tf}}+\frac{1}{3} \gamma_{i j} R .
$$

Here, "f denotes the trace-free part of a spatial tensor defined by $X_{i j}^{\mathrm{tf}}=X_{i j}-\frac{1}{3} \gamma_{i j} X$ with trace $X=\gamma^{k l} X_{k l}$. Our convention for the Ricci tensor is as follows:

$$
R_{i j}=\partial_{k} \Gamma^{k}{ }_{i j}-\partial_{i} \Gamma^{k}{ }_{j k}+\Gamma^{k}{ }_{k l} \Gamma^{l}{ }_{i j}-\Gamma^{k}{ }_{i l} \Gamma^{l}{ }_{j k},
$$

where $\Gamma^{k}{ }_{i j}$ is the Christoffel symbol associated with the spatial metric $\gamma_{i j}$. In the following we use as dynamical variables $A_{i j}, K$, the three-metric $\gamma_{i j}$, the scalar field $\Phi$, and the scalar field momentum $K_{\Phi}$.

Decomposition of the Weyl tensor: We define the gravitoelectric and gravitomagnetic components of the Weyl tensor $W_{a b c d}$,

$$
\begin{gathered}
E_{i j}=\gamma^{a}{ }_{i} \gamma^{b}{ }_{j} n^{c} n^{d} W_{a c b d}, \\
B_{i j}=\gamma^{a}{ }_{i} \gamma^{b}{ }_{j} n^{c} n^{d *} W_{a c b d},
\end{gathered}
$$

respectively, where ${ }^{*} W_{a b c d}$ denotes the dual Weyl tensor. The Weyl tensor can be expressed in terms of $E_{i j}$ and $B_{i j}$ as [49]

$$
\begin{aligned}
W_{a b c d}= & 2\left(l_{a[c} E_{d] b}-l_{b[c} E_{d] a}\right. \\
& \left.-n_{[c} B_{d] e} \epsilon_{a b}^{e}-n_{[a} B_{b] e} \epsilon_{c d}^{e}\right),
\end{aligned}
$$

where $\epsilon_{i j k}$ is the three-dimensional Levi-Civita tensor $\left(\epsilon_{a b c}=n^{d} \epsilon_{d a b c}\right)$, and

$$
l_{a b}=g_{a b}+2 n_{a} n_{b}=\gamma_{a b}+n_{a} n_{b} .
$$

Note that

$$
W_{a b c d} W^{a b c d}=8\left(E^{i j} E_{i j}-B^{i j} B_{i j}\right)
$$

which contributes to the GB invariant $\mathcal{R}_{\mathrm{GB}}$; see Eq. (7a).

We now derive a geometric relation between the extrinsic curvature and the gravitoelectric and gravitomagnetic fields defined in Eqs. (15). Therefore, we decompose the Weyl tensor in terms of the $3+1$ variables, i.e., in terms of the 3metric and extrinsic curvature. By inserting the resulting expression in Eqs. (15) we find 


$$
E_{i j}=\frac{1}{2}\left(\mathcal{L}_{n} A_{i j}+R_{i j}^{\mathrm{tf}}+\frac{1}{\alpha}\left[D_{i} D_{j} \alpha\right]^{\mathrm{tf}}\right)+\frac{1}{3} \gamma_{i j} A^{2}+\frac{1}{6} K A_{i j},
$$

$$
B_{i j}=\epsilon_{(i \mid}^{k l} D_{k} A_{\mid j)^{l}},
$$

where $A^{2} \equiv A^{k l} A_{k l}$. Note that these relations are of purely geometric origin and so are independent of the specific field equations. By construction the gravitoelectric and gravitomagnetic parts of the Weyl tensor are trace-free and spatial, i.e., $\gamma^{i j} E_{i j}=0, E_{a b} n^{a}=0$, and likewise for $B_{i j}$.

Auxiliary tensors: To write the sGB constraint and evolution equations in a compact form, we may find it useful to employ quantities obtained in vacuum GR as shorthand. In particular, we introduce

$$
\begin{aligned}
\mathcal{H}^{\mathrm{GR}} & =2 G_{a b} n^{a} n^{b}=R-A^{2}+\frac{2}{3} K^{2}, \\
\mathcal{M}_{i}^{\mathrm{GR}} & =-\gamma^{a}{ }_{i} n^{b} G_{a b}=D^{k} A_{i k}-\frac{2}{3} D_{i} K .
\end{aligned}
$$

Note that $\mathcal{H}^{\mathrm{GR}}=0$ and $\mathcal{M}_{i}^{\mathrm{GR}}=0$ are the constraint equations in vacuum GR. We find it useful to employ the expression for the gravitoelectric field in vacuum GR as shorthand. Therefore we take the geometric, i.e., theoryindependent, relation (19a) and substitute $\mathcal{L}_{n} A_{i j}$ with the corresponding dynamical (i.e., theory-dependent) evolution equation obtained in GR. That is, we insert

$$
\mathcal{L}_{n} A_{i j}=-\frac{1}{\alpha}\left[D_{i} D_{j} \alpha\right]^{\mathrm{tf}}+R_{i j}^{\mathrm{tf}}-2 A_{i k} A^{k}{ }_{j}+\frac{1}{3} K A_{i j}
$$

into Eq. (19a), and we find

$$
E_{i j}^{\mathrm{GR}}=R^{\mathrm{tf}}-A_{i k} A_{j}^{k}+\frac{1}{3} K A_{i j}+\frac{1}{3} \gamma_{i j} A^{2} .
$$

Note that $\gamma^{i j} E_{i j}^{\mathrm{GR}}=0$.

\section{Kinematic evolution equations}

The geometric relations (10), (11) determine the kinematic evolution equations for the spatial metric and scalar field. In terms of the dynamical variables defined in Sec. III B they are given by

$$
\begin{aligned}
\mathrm{d}_{t} \Phi & =-\alpha K_{\Phi}, \\
\mathrm{d}_{t} \gamma_{i j} & =-2 \alpha\left(A_{i j}+\frac{1}{3} \gamma_{i j} K\right),
\end{aligned}
$$

where $\mathrm{d}_{t} \equiv \partial_{t}-\mathcal{L}_{\beta}$. We remark that Eqs. (23) are not affected by the GB coupling: the kinematic evolution equations in SGB gravity coincide with those of GR (with a minimally coupled scalar field). This is because the latter decomposition is of purely geometric nature and therefore viable for any metric theory of gravity. The dynamics of a specific theory are determined by its field equations. To derive the time evolution formulation of sGB gravity, we need to apply the spacetime split introduced in Sec. III A to the equations of motion (3).

\section{Constraints}

We obtain the constraint equations of sGB gravity by contracting the tensor field equations ( $3 \mathrm{~b}$ ) with the normal vector $n^{a}$. The modified Hamiltonian constraint becomes

$$
\begin{aligned}
\mathcal{H}= & \mathcal{H}^{\mathrm{GR}}\left(1-\frac{\alpha_{\mathrm{GB}}}{3} C\right)-\frac{1}{2}\left(K_{\Phi}^{2}+D^{k} \Phi D_{k} \Phi\right) \\
& +2 \alpha_{\mathrm{GB}} E_{k l}^{\mathrm{GR}} C^{\mathrm{ff} \mathrm{kl}},
\end{aligned}
$$

where $C=\gamma^{i j} C_{i j}$ and $C_{i j}^{\mathrm{tf}}$ denotes the trace-free part of the spatial scalar tensor $C_{i j}$ given in Eqs. (12). The momentum constraint is given by

$$
\begin{aligned}
\mathcal{M}_{i}= & \mathcal{M}_{i}^{\mathrm{GR}}-\frac{1}{2} K_{\Phi} D_{i} \Phi+\alpha_{\mathrm{GB}} E_{i j}^{\mathrm{GR}} C^{j}-\frac{\alpha_{\mathrm{GB}}}{6} \mathcal{H}^{\mathrm{GR}} C_{i} \\
& +\alpha_{\mathrm{GB}}\left(C^{j}{ }_{[i} \mathcal{M}_{j]}^{\mathrm{GR}}-\epsilon_{i j k} C^{j}{ }_{l} B^{k l}\right),
\end{aligned}
$$

where the scalar tensors have been defined in Eqs. (12) and $\mathcal{H}^{\mathrm{GR}}$ and $\mathcal{M}_{i}^{\mathrm{GR}}$ are given in Eqs. (20). In the limit $\alpha_{\mathrm{GB}} \rightarrow 0$ the constraints (24) and (25) reduce to those of GR minimally coupled to a scalar field.

\section{E. Dynamical evolution equations}

We obtain the dynamical, i.e., model-dependent, evolution equations by decomposing the scalar field equation (3a) and by fully projecting the tensor field equations ( $3 b)$ on the spatial hypersurfaces. Here, we express equations in terms of the Lie derivative along the normal vector; we remind the reader that it is related to the time derivative via $\mathcal{L}_{n} X=\frac{1}{\alpha} \mathrm{d}_{t} X=\frac{1}{\alpha}\left(\partial_{t}-\mathcal{L}_{\beta}\right) X$.

The scalar field momentum $K_{\Phi}$ evolves according to

$$
\begin{aligned}
0= & -\mathcal{L}_{n} K_{\Phi}-D^{k} D_{k} \Phi+K K_{\Phi}-\frac{1}{\alpha} D^{k} \Phi D_{k} \alpha \\
& -\frac{\alpha_{\mathrm{GB}}}{4} f^{\prime} \mathcal{R}_{\mathrm{GB}},
\end{aligned}
$$

where the GB invariant can be written as

$$
\begin{aligned}
\mathcal{R}_{\mathrm{GB}}= & -\frac{4}{3} \mathcal{H}^{\mathrm{GR}}\left[\mathcal{L}_{n} K+\frac{1}{\alpha} D^{k} D_{k} \alpha-A^{2}-\frac{1}{3} K^{2}\right] \\
& +8 E^{\mathrm{GR} \mathrm{kl}}\left[\mathcal{L}_{n} A_{k l}+\frac{1}{\alpha}\left[D_{i} D_{j} \alpha\right]^{\mathrm{tf}}+A_{j k} A^{j}{ }_{l}\right] \\
& -8 B_{k l} B^{k l}+4 \mathcal{M}_{k}^{\mathrm{GR}} \mathcal{M}^{\mathrm{GR}},
\end{aligned}
$$

in terms of the Arnowitt-Deser-Misner variables. 
The time evolution of the trace of the extrinsic curvature is determined by

$$
\begin{aligned}
0= & -\left(1-\frac{\alpha_{\mathrm{GB}}}{3} C\right)\left[\mathcal{L}_{n} K+\frac{1}{\alpha} D^{k} D_{k} \alpha-A^{2}-\frac{1}{3} K^{2}\right] \\
& -\frac{\alpha_{\mathrm{GB}}}{2} C^{\mathrm{tf} \mathrm{kl}} \mathcal{L}_{n} A_{k l}+\frac{1}{2} K_{\Phi}^{2} \\
& -\frac{\alpha_{\mathrm{GB}}}{2} C^{\mathrm{tf} \mathrm{kl}}\left[E_{k l}^{\mathrm{GR}}+\frac{1}{\alpha}\left[D_{k} D_{l} \alpha\right]^{\mathrm{tf}}+A_{k j} A^{j} l\right] \\
& +\frac{\alpha_{\mathrm{GB}}}{4} \mathcal{H}^{\mathrm{GR}}\left(C_{\mathrm{nn}}+\frac{1}{3} C\right)-\alpha_{\mathrm{GB}} \mathcal{M}_{k}^{\mathrm{GR}} C^{k},
\end{aligned}
$$

where we have used the Hamiltonian constraint (24). The auxiliary variable $C_{\mathrm{nn}}$, defined in Eq. (12a), can be rewritten in terms of the GB invariant as

$C_{\mathrm{nn}}=C+\frac{\alpha_{\mathrm{GB}}}{4}\left(f^{\prime}\right)^{2} \mathcal{R}_{\mathrm{GB}}+f^{\prime \prime}\left(K_{\Phi}^{2}-D^{k} \Phi D_{k} \Phi\right)$

where $C=\gamma^{i j} C_{i j}$ is the trace of the spatial scalar field tensor given in Eq. (12c) and we inserted the evolution equation (26). That is, we have traded terms $\sim \mathcal{L}_{n} K_{\Phi}$ with terms $\sim\left(\mathcal{L}_{n} K, \mathcal{L}_{n} A_{i j}\right)$ "hidden" in the GB invariant (27).
Finally, comparing the spatial projection of the tensor field equations (3b) with the geometric relation (19a) yields the evolution equation for $A_{i j}$,

$$
\begin{aligned}
0= & -H_{i j}{ }^{k l}\left[\mathcal{L}_{n} A_{k l}+\frac{1}{\alpha}\left[D_{i} D_{j} \alpha\right]^{\mathrm{tf}}+A_{k m} A^{m}{ }_{l}\right] \\
& +\frac{\alpha_{\mathrm{GB}}}{3} C_{i j}^{\mathrm{ff}}\left[\mathcal{L}_{n} K+\frac{1}{\alpha} D^{k} D_{k} \alpha-3 A^{2}-\frac{1}{3} K^{2}\right] \\
& -\frac{1}{2}\left[D_{i} D_{j} \Phi\right]^{\mathrm{tf}}+\left(1+\alpha_{\mathrm{GB}} C_{\mathrm{nn}}\right) E_{i j}^{\mathrm{GR}} \\
& -\alpha_{\mathrm{GB}}\left[\mathcal{M}_{(i}^{\mathrm{GR}} C_{j)}\right]^{\mathrm{tf}}-2 \alpha_{\mathrm{GB}} \epsilon_{(i}{ }^{k l} B_{j) k} C_{l} .
\end{aligned}
$$

Here, we introduced the operator

$$
H^{i j k l}=\gamma^{k(i} \mathcal{F}^{j) l}-\frac{1}{3} \gamma^{i j} \mathcal{F}^{k l},
$$

with

$$
\mathcal{F}_{i j}=\left(1-\frac{\alpha_{\mathrm{GB}}}{3} C\right) \gamma_{i j}+2 \alpha_{\mathrm{GB}} C_{i j}^{\mathrm{tf}} .
$$

The system of equations (26), (28), and (30) is still coupled in a nontrivial way. Therefore, we write it in matrix form and analyze the resulting coefficient matrix in detail. Specifically we obtain

$$
\left(\begin{array}{ccc}
1 & -\frac{\alpha_{\mathrm{GB}}}{3} f^{\prime} \mathcal{H}^{\mathrm{GR}} & 2 \alpha_{\mathrm{GB}} f^{\prime} E^{\mathrm{GR} \mathrm{kl}} \\
0 & 1-\frac{\alpha_{\mathrm{GB}}}{3} C+\frac{\alpha_{\mathrm{GB}}^{2}}{12} f^{\prime 2}\left(\mathcal{H}^{\mathrm{GR}}\right)^{2} & \frac{\alpha_{\mathrm{GB}}}{2} C^{\mathrm{ff} \mathrm{kl}}-\frac{\alpha_{\mathrm{GB}}^{2}}{2} f^{\prime 2} \mathcal{H}^{\mathrm{GR}} E^{\mathrm{GR} \mathrm{kl}} \\
0 & -\frac{\alpha_{\mathrm{GB}}}{3} C_{i j}^{\mathrm{ff}}+\frac{\alpha_{\mathrm{GB}}^{2}}{3} f^{\prime 2} \mathcal{H}^{\mathrm{GR}} E_{i j}^{\mathrm{GR}} & H_{i j}{ }^{k l}-2 \alpha_{\mathrm{GB}}^{2} f^{\prime 2} E_{i j}^{\mathrm{GR}} E^{\mathrm{GR} \mathrm{kl}}
\end{array}\right)\left(\begin{array}{c}
\mathcal{L}_{n} K_{\Phi} \\
\mathcal{L}_{n} K \\
\mathcal{L}_{n} A_{k l}
\end{array}\right)=\left(\begin{array}{c}
\mathcal{S}^{\Phi} \\
\mathcal{S}^{K} \\
\mathcal{S}_{i j}^{\mathrm{A}}
\end{array}\right),
$$

where the (time independent) source terms are given by

$$
\begin{aligned}
& \mathcal{S}^{\Phi}=-D^{i} D_{i} \Phi+K K_{\Phi}-\frac{1}{\alpha} D^{i} \alpha D_{i} \Phi+\frac{\alpha_{\mathrm{GB}}}{3} f^{\prime} \mathcal{H}^{\mathrm{GR}}\left(\frac{1}{\alpha} D^{i} D_{i} \alpha-A^{2}-\frac{1}{3} K^{2}\right) \\
& -2 \alpha_{\mathrm{GB}} f^{\prime} E^{\mathrm{GR} \mathrm{kl}}\left(\frac{1}{\alpha}\left[D_{i} D_{j} \alpha\right]^{\mathrm{tf}}+A_{k j} A^{j}{ }_{l}\right)+\alpha_{\mathrm{GB}} f^{\prime}\left(2 B_{k l} B^{k l}-\mathcal{M}_{k}^{\mathrm{GR}} \mathcal{M}^{\mathrm{GR} \mathrm{k}}\right), \\
& \mathcal{S}^{K}=-\left(1-\frac{\alpha_{\mathrm{GB}}}{3} C+\frac{\alpha_{\mathrm{GB}}^{2}}{12} f^{\prime 2}\left(\mathcal{H}^{\mathrm{GR}}\right)^{2}\right)\left(\frac{1}{\alpha} D^{k} D_{k} \alpha-A^{2}-\frac{1}{3} K^{2}\right)+\frac{1}{2} K_{\Phi}^{2}+\frac{\alpha_{\mathrm{GB}}}{4} f^{\prime \prime} \mathcal{H}^{\mathrm{GR}}\left(K_{\Phi}^{2}-D^{k} \Phi D_{k} \Phi\right) \\
& -\frac{1}{2}\left(\alpha_{\mathrm{GB}} C^{\mathrm{tf} \mathrm{kl}}+\alpha_{\mathrm{GB}}^{2} f^{\prime 2} \mathcal{H}^{\mathrm{GR}} E^{\mathrm{GR} \mathrm{kl}}\right)\left(\frac{1}{\alpha}\left[D_{k} D_{l} \alpha\right]^{\mathrm{tf}}+A_{k m} A^{m}{ }_{l}\right)-\frac{\alpha_{\mathrm{GB}}^{2}}{4} f^{\prime 2} \mathcal{H}^{\mathrm{GR}}\left(2 B^{k l} B_{k l}-\mathcal{M}^{\mathrm{GR} \mathrm{k}} \mathcal{M}_{k}^{\mathrm{GR}}\right) \\
& -\alpha_{\mathrm{GB}}\left(C^{k} \mathcal{M}_{k}^{\mathrm{GR}}-\frac{1}{3} C \mathcal{H}^{\mathrm{GR}}+\frac{1}{2} C^{\mathrm{tf} k \mathrm{l}} E_{k l}^{\mathrm{GR}}\right) \text {, } \\
& \left.\mathcal{S}_{i j}^{A}=\left(\frac{\alpha_{\mathrm{GB}}}{3} C_{i j}^{\mathrm{tf}}-\frac{\alpha_{\mathrm{GB}}^{2}}{3} f^{\prime 2} \mathcal{H}^{\mathrm{GR}} E_{i j}^{\mathrm{GR}}\right)\left(\frac{1}{\alpha} D^{k} D_{k} \alpha-A^{2}-\frac{1}{3} K^{2}\right)+2 \alpha_{\mathrm{GB}} \epsilon_{(i}{ }^{k l} B_{j) k} C_{l}-\alpha_{\mathrm{GB}}\left[\mathcal{M}_{(i}^{\mathrm{GR}} C_{j)}\right]\right]^{\mathrm{tf}} \\
& -\left(H_{i j}{ }^{k l}-2 \alpha_{\mathrm{GB}}^{2} f^{\prime 2} E_{i j}^{\mathrm{GR}} E^{\mathrm{GR} \mathrm{kl}}\right)\left(\frac{1}{\alpha}\left[D_{k} D_{l} \alpha\right]^{\mathrm{tf}}+A_{k m} A^{m}{ }_{l}+\frac{1}{3} \gamma_{k l} A^{2}\right) \\
& -\frac{1}{2}\left[D_{i} D_{j} \Phi\right]^{\mathrm{tf}}+E_{i j}^{\mathrm{GR}}\left(1+\alpha_{\mathrm{GB}} C+\alpha_{\mathrm{GB}} f^{\prime \prime}\left[K_{\Phi}^{2}-D^{k} \Phi D_{k} \Phi\right]\right)-\alpha_{\mathrm{GB}}^{2} f^{\prime 2} E_{i j}^{\mathrm{GR}}\left(2 B^{k l} B_{k l}-\mathcal{M}^{\mathrm{GRk}} \mathcal{M}_{k}^{\mathrm{GR}}\right) \text {. }
\end{aligned}
$$


The above system of dynamical equations, supplemented by the constraint equations (24) and (25), is one of the main results of this work.

\section{ON THE FORMULATION OF THE EVOLUTION EQUATIONS}

A time evolution of the dynamical equations of sGB gravity should first of all be well formulated; i.e., it should be written as a system of first-order-in-time field equations. In addition, it should be well posed; i.e., there should be a unique solution with continuous dependence on given initial data. In this section we shall discuss separately these two requirements.

\section{A. Looking for a well-formulated set of equations}

Equations (33) form a linear system in $\left(\mathcal{L}_{n} K_{\Phi}, \mathcal{L}_{n} K\right.$, $\left.\mathcal{L}_{n} A_{k l}\right)$, i.e., in the Lie derivatives along the normal vector (describing the time evolution) of the dynamical variables $K_{\Phi}, K$, and $A_{i j}$. The Lie derivatives of the other dynamical variables, $\Phi$ and $\gamma_{i j}$, are given by the kinematical evolution equations discussed in Sec. III C. The matrix components and the source terms in Eq. (33), instead, depend on the entire set of dynamical variables $\left\{\Phi, \gamma_{i j}, K_{\Phi}, K, A_{k l}\right\}$ and on their space derivatives.

The form (33) is not appropriate for a well-formulated time evolution problem, because the components of the vector $\left(\mathcal{L}_{n} K_{\Phi}, \mathcal{L}_{n} K, \mathcal{L}_{n} A_{k l}\right)$ are not independent. Indeed, the symmetric and traceless tensor $A_{i j}$ has nine components, but only five of them are independent. Note that $\mathcal{L}_{n} A_{k l}$ is symmetric but not traceless; however, it has five independent components due to the relation $\gamma^{k l} \mathcal{L}_{n} A_{k l}=$ $-2 A^{2}$ [see Eq. (19a)]. Therefore the trace-free part of $\mathcal{L}_{n} A_{i j}$ is given by

$$
\mathcal{L}_{n} A_{i j}=\mathcal{L}_{n} A_{i j}{ }^{\mathrm{tf}}-\frac{2}{3} \gamma_{k l} A^{2} .
$$

Note also that $\mathcal{L}_{n} A_{i j}$ appears in Eq. (33) multiplied by the trace-free tensors $C_{i j}^{\mathrm{tf}}, E_{i j}^{\mathrm{GR}}$, and $H_{i j}{ }^{k l}$; therefore only its trace-free part, $\mathcal{L}_{n} A_{i j}{ }^{\text {ff }}$, contributes to Eq. (33).

To extract a set of independent degrees of freedom, we decompose $A_{i j}$ and $\mathcal{L}_{n} A_{i j}{ }^{\text {tf }}$ in a basis of symmetrictrace-free (STF) tensors as suggested in [50] (see also [51],
Chap. 1). Following the notation of [50], we denote the components of an $l$ th rank STF tensor $T_{i_{1} \cdots i_{l}}$ as $T_{\langle L\rangle}$. For $l=2, T_{\langle L\rangle}=T_{i j}$ symmetric and traceless, and (we remind the reader that repeated indices $i, j, \ldots$ are summed from 1 to 3 )

$$
T_{2 m}=\mathcal{Y}_{i j 2 m} T_{i j} \quad T_{i j}=N_{2} \sum_{m=-2}^{2} \mathcal{Y}_{i j 2 m}^{*} T_{2 m},
$$

$N_{l}=4 \pi l ! /(2 l+1) ! !$ and

$$
\mathcal{Y}_{i j 2 m}=N_{2}^{-1} \int z^{\langle i j\rangle} Y_{2 m}^{*}(\theta, \phi) d \Omega,
$$

where $\quad z^{\langle i j\rangle}=z^{\langle i} z^{j\rangle}=z^{(i} z^{j)}-\frac{1}{3} \delta^{i j}, \quad z^{i}=(\sin \theta \cos \phi$, $\sin \theta \sin \phi, \cos \theta)$, * denotes complex conjugation, and $Y_{l m}(\theta, \phi)$ are the spherical harmonics.

Therefore, defining the variables $A_{m}$ and $\mathcal{L}_{n} A_{2 m}$ (with five independent components for $m=-2, \ldots, 2$ each) through $^{1}$

$$
\begin{aligned}
A_{k l} & =N_{2} \mathcal{Y}_{k l 2 m}^{*} A_{2 m}, \\
\mathcal{L}_{n} A_{k l}^{\mathrm{tf}} & =N_{2} \mathcal{Y}_{k l 2 m}^{*} \mathcal{L}_{n} A_{2 m},
\end{aligned}
$$

i.e.,

$$
\begin{aligned}
A_{2 m} & =\mathcal{Y}_{k l 2 m} A_{k l}, \\
\mathcal{L}_{n} A_{2 m} & =\mathcal{Y}_{k l 2 m} \mathcal{L}_{n} A_{k l}{ }^{\mathrm{tf}},
\end{aligned}
$$

and defining

$$
\begin{aligned}
E_{m}^{\mathrm{GR}} & =N_{2} \mathcal{Y}_{k l 2 m}^{*} E^{\mathrm{GR} k l}, \\
\mathcal{C}_{m} & =N_{2} \mathcal{Y}_{k l 2 m}^{*} \mathcal{C}^{\mathrm{tf} k l}, \\
S_{m^{\prime}}^{A} & =N_{2} \mathcal{Y}_{i j 2 m^{\prime}} S^{A i j}, \\
H_{m m^{\prime}} & =N_{2}^{2} \mathcal{Y}_{i j 2 m^{\prime}} \mathcal{Y}_{k l 2 m}^{*} H^{i j k l},
\end{aligned}
$$

the field equations (33) reduce to the $7 \times 7$ system

$$
\mathbb{M}\left(\begin{array}{c}
\mathcal{L}_{n} K_{\Phi} \\
\mathcal{L}_{n} K \\
\mathcal{L}_{n} A_{2 m}
\end{array}\right)=\left(\begin{array}{c}
\mathcal{S}^{\Phi} \\
\mathcal{S}^{K} \\
\mathcal{S}_{m^{\prime}}^{A}
\end{array}\right)
$$

where

$$
\mathbb{M}=\left(\begin{array}{cc|c}
1 & -\frac{1}{3} \alpha_{\mathrm{GB}} f^{\prime} \mathcal{H}^{\mathrm{GR}} & 2 \alpha_{\mathrm{GB}} f^{\prime} E_{m}^{\mathrm{GR}} \\
0 & 1-\frac{\alpha_{\mathrm{GB}}}{3} C+\frac{\alpha_{\mathrm{GB}}^{2}}{12} f^{\prime 2}\left(\mathcal{H}^{\mathrm{GR}}\right)^{2} & \frac{\alpha_{\mathrm{GB}}}{2} \mathcal{C}_{m}-\frac{\alpha_{\mathrm{GB}}^{2}}{2} f^{\prime 2} \mathcal{H}^{\mathrm{GR}} E_{m}^{\mathrm{GR}} \\
\hline 0 & -\frac{\alpha_{\mathrm{GB}}}{3} \mathcal{C}_{m^{\prime}}^{*}+\frac{\alpha_{\mathrm{GB}}^{2}}{3} f^{\prime 2} \mathcal{H}^{\mathrm{GR}} E_{m^{\prime}}^{\mathrm{GR} *} & H_{m m^{\prime}}-2 \alpha_{\mathrm{GB}}^{2} f^{\prime 2} E_{m}^{\mathrm{GR}} E_{m^{\prime}}^{\mathrm{GR} *}
\end{array}\right)
$$

\footnotetext{
${ }^{1}$ Note that the quantities $\mathcal{L}_{n} A_{2 m}$ are the (trace-free) Lie derivative of a rank-two tensor, projected on the $l=2$ spherical harmonics.
} 
is a seven-dimensional square matrix. Note that, to obtain Eq. (42) from the matrix in Eq. (33), we have raised the indices $(i, j)$ and multiplied the bottom lines with $N_{2} \mathcal{Y}_{i j 2 m^{\prime}}$. The system (41) is one of the main results of this work.

This is a system of seven first-order-in-time differential equations in terms of the seven independent variables $\left\{K_{\Phi}, K, A_{2 m}\right\}$. A well-formulated system of field equations would have the form

$$
\left(\begin{array}{c}
\mathcal{L}_{n} K_{\Phi} \\
\mathcal{L}_{n} K \\
\mathcal{L}_{n} A_{2 m}
\end{array}\right)=\mathbb{M}^{-1}\left(\begin{array}{c}
\mathcal{S}^{\Phi} \\
\mathcal{S}^{K} \\
\mathcal{S}_{m^{\prime}}^{A}
\end{array}\right)
$$

Thus, we can write a well-formulated time evolution of the sGB field equations if and only if the matrix $\mathbb{M}$ is invertible, i.e., if

$$
\operatorname{det}(\mathbb{M}) \neq 0
$$

along any physically significant evolution.

We cannot prove that Eq. (44) is always satisfied. However, we have an indication that this may be the case. Indeed, let us compare sGB gravity with a different quadratic gravity theory, dynamical Chern-Simons gravity (see, e.g., [52] and references therein). In that case, as noted in [21] [see their Eqs. (41) and (54)], the evolution equation for the auxiliary variable $X_{i j}$ (related to $\mathcal{L}_{n} A_{i j}$ ) has the form

$$
\partial_{t}\left(\delta_{(i}^{(k} \epsilon_{j)}^{l) m}\left(D_{m} \Phi\right) X_{k l}\right)=S_{i j},
$$

which is necessarily degenerate due to the presence of the Levi-Civita tensor. These terms have been a key obstruction in inverting the coefficient matrix in dynamical Chern-Simons gravity. They are absent in sGB gravity, so one of the core bottlenecks is absent and one might be able to find an inversion for $\mathbb{M}$. Of course, this does prevent other, different obstructions to be present in sGB gravity.

Finally, note that $\mathbb{M}$ is invertible if sGB gravity is treated perturbatively. In this case it is sufficient to require that $\mathbb{M}$ is invertible at zeroth order, since $\operatorname{det}(\mathbb{M})$ can be expanded in the coupling constant and, if it does not vanish to zeroth order, it cannot change sign due to perturbative corrections. Owing to the simplified block-diagonal form of Eq. (42) in the $\alpha_{\mathrm{GB}} \rightarrow 0$ limit, the requirement that $\operatorname{det}(\mathbb{M}) \neq 0$ to zeroth order reduces to the invertibility of the $5 \times 5$ submatrix $H_{m m^{\prime}}$. Since, from Eq. (31),

$$
H^{i j k l}=\gamma^{k(i} \gamma^{j) l}-\frac{1}{3} \gamma^{i j} \gamma^{k l} \quad \text { when } \alpha_{\mathrm{GB}}=0,
$$

we obtain

$$
H_{m m^{\prime}}=N_{2}^{2} \mathcal{Y}^{k l}{ }_{2 m^{\prime}} \mathcal{Y}_{k l 2 m}^{*}=\delta_{m m^{\prime}}
$$

Thus, to zeroth order in $\alpha_{\mathrm{GB}}$, the $\mathbb{M}$ matrix reduces to the identity matrix, which is trivially invertible and constant in time.

Beyond the small-coupling limit, it is tempting to conjecture that if $\mathbb{M}$ is invertible at $t=0$, it must be so during the evolution as a consequence of the field equations. We were not able to prove such a statement and its (dis)proof is left for future work.

\section{B. On the well-posedness of sGB gravity}

Once the field equations of sGB gravity are written as a well-formulated time evolution problem-i.e., assuming the matrix $\mathbb{M}$ presented in Eq. (42) is invertible-the next step is to look for a well-posed formulation. That is, one would typically attempt to express the field equations as a strongly hyperbolic system. A full hyperbolicity analysis is beyond the scope of this paper. However, it is useful to look at the structure of the equations by identifying their highest derivative terms. Inspection of Eqs. (33) and (41) shows the presence of terms such as

$$
E_{i j}^{\mathrm{GR}} E_{k l}^{\mathrm{GR}} \sim R_{i j}^{\mathrm{ff}} R_{k l}^{\mathrm{ff}}, \quad \mathcal{H}^{\mathrm{GR}} E_{i j}^{\mathrm{GR}} \sim R R_{i j}^{\mathrm{tf}},
$$

which are quadratic in the second spatial derivatives of the spatial metric. They are present both in the coefficient matrix (42) and in the source terms (34c). These terms are nonlinear and can, therefore, spoil the strong hyperbolicity of the system, leading to characteristic crossing (and thus multivalued dependence on the initial data) or to a change of the character of the equations in different spacetime regions, as shown in [38] in the spherically symmetric case.

We remark, however, that the existence of such a term does not rule out the possibility of a well-posed formulation. For instance, in the case of cubic Horndeski gravity a strongly hyperbolic formulation has been found [30] despite the presence of terms quadratic in the second spatial derivatives [see, e.g., Eq. (107) of [30] ]. A similar analysis in sGB gravity will be the subject of a forthcoming publication.

\section{CONCLUSIONS AND OUTLOOK}

We have presented the $3+1$ decomposition of the field equations in sGB gravity, writing them as a set of evolution equations and of elliptic constraints. This work is only the first step toward evolving black-hole binaries in sGB gravity and could be useful for a general proof of wellposedness of this theory.

The initial-value problem for this theory is significantly more involved than in GR. We managed to recast the standard $3+1$ system of equations into a seven-dimensional first-order-in-time system of equations for seven independent dynamical variables [cf. Eq. (41)]. This requires the inversion of a seven-dimensional matrix written in terms of the dynamical variables and their spatial derivatives. We have 
proved the invertibility of this matrix in the small-coupling limit, and we have found indications that it should also be invertible for finite values of the coupling (at variance with other theories, such as dynamical Chern-Simons gravity [21]). A complete proof of the invertibility of this matrix (i.e., of the existence of a well-formulated time evolution) is left for future work.

The derived field equations contain nonlinear terms, quadratic in the second spatial derivatives of the spatial metric, which can spoil the strong hyperbolicity of the system. However, such terms do not necessarily prevent a well-posed formulation, which should therefore be analyzed in detail.

We also derived the explicit form of the constraint equations for sGB with a generic coupling function. These (elliptic) equations are significantly more involved than in the GR case. Future work will also focus on finding approximated or numerical solutions to the constraints equations, to be used in simulations of black-hole binaries in nonperturbative sGB gravity.

\section{ACKNOWLEDGMENTS}

We thank F. Julié and T. Sotiriou for useful discussions. H. W. acknowledges financial support provided by the Royal Society University Research Fellowship
UF160547 and the Royal Society Research Grant No. RGF\R1\180073. P. P. acknowledges financial support provided under the European Union's H2020 ERC, Starting Grant Agreement No. DarkGRA-757480, under the MIUR PRIN and FARE programs (GWNEXT, CUP: B84I20000100001), and support from the Amaldi Research Center funded by the MIUR program "Dipartimento di Eccellenza" (CUP: B81I18001170001). We thankfully acknowledge the computer resources and the technical support provided by the Leibniz Supercomputing Center via PRACE Grant No. 2018194669 "FunPhysGW: Fundamental Physics in the era of gravitational waves" and by the DiRAC Consortium via STFC DiRAC Grants No. ACTP186, No. ACSP191 and No. ACSP218. The authors would like to acknowledge networking support by the COST Action CA16104. The XTENSOR package for Mathematica $[53,54]$ has been used.

Note added.-While this work was near completion, we discovered a related work by Félix-Louis Julié and Emanuele Berti [55], appearing on the same day, which focuses on the Hamiltonian formulation of SGB gravity. The overall conclusions of this work for what concerns the time evolution of the theory are in agreement with ours.
[1] B. P. Abbott et al. (LIGO Scientific, Virgo Collaborations), Phys. Rev. Lett. 116, 221101 (2016); 121, 129902(E) (2018).

[2] E. Berti et al., Classical Quantum Gravity 32, 243001 (2015).

[3] K. Yagi and L. C. Stein, Classical Quantum Gravity 33, 054001 (2016).

[4] N. Yunes and X. Siemens, Living Rev. Relativity 16, 9 (2013).

[5] L. Barack et al., Classical Quantum Gravity 36, 143001 (2019).

[6] M. Agathos, W. Del Pozzo, T. G. F. Li, C. Van Den Broeck, J. Veitch, and S. Vitale, Phys. Rev. D 89, 082001 (2014).

[7] N. Yunes and F. Pretorius, Phys. Rev. D 80, 122003 (2009).

[8] E. Berti, K. Yagi, and N. Yunes, Gen. Relativ. Gravit. 50, 46 (2018).

[9] E. Berti, K. Yagi, H. Yang, and N. Yunes, Gen. Relativ. Gravit. 50, 49 (2018).

[10] M. Okounkova, L. C. Stein, M. A. Scheel, and D. A. Hemberger, Phys. Rev. D 96, 044020 (2017).

[11] H. Witek, L. Gualtieri, P. Pani, and T. P. Sotiriou, Phys. Rev. D 99, 064035 (2019).

[12] M. Okounkova, L. C. Stein, M. A. Scheel, and S. A. Teukolsky, Phys. Rev. D 100, 104026 (2019).

[13] M. Okounkova, arXiv:2001.03571.

[14] E. Barausse, C. Palenzuela, M. Ponce, and L. Lehner, Phys. Rev. D 87, 081506 (2013).
[15] C. Palenzuela, E. Barausse, M. Ponce, and L. Lehner, Phys. Rev. D 89, 044024 (2014).

[16] M. Shibata, K. Taniguchi, H. Okawa, and A. Buonanno, Phys. Rev. D 89, 084005 (2014).

[17] D. D. Doneva and S. S. Yazadjiev, Phys. Rev. Lett. 120, 131103 (2018).

[18] H. O. Silva, J. Sakstein, L. Gualtieri, T. P. Sotiriou, and E. Berti, Phys. Rev. Lett. 120, 131104 (2018).

[19] R. P. Woodard, Lect. Notes Phys. 720, 403 (2007).

[20] D. Hilditch, Int. J. Mod. Phys. A 28, 1340015 (2013).

[21] T. Delsate, D. Hilditch, and H. Witek, Phys. Rev. D 91, 024027 (2015).

[22] H. Ringström, The Cauchy Problem in General Relativity (ESI Lectures in Mathematics and Physics, European Mathematical Society, Berlin, 2009).

[23] Y. Choquet-Bruhat, arXiv:1410.3490.

[24] O. Sarbach and M. Tiglio, Living Rev. Rel. 15, 9 (2012).

[25] M. Salgado, D. Martinez-del Rio, M. Alcubierre, and D. Nunez, Phys. Rev. D 77, 104010 (2008).

[26] M. Salgado, Classical Quantum Gravity 23, 4719 (2006).

[27] O. Sarbach and E. Barausse, and J. A Preciado-López, Class. Quant. Grav. 16, 165007 (2019).

[28] A. D. Kovacs and H. S. Reall, Phys. Rev. Lett. 124, 221101 (2020).

[29] A. D. Kovacs and H. S. Reall, Phys. Rev. D 101, 124003 (2020). 
[30] A. D. Kovacs, Phys. Rev. D 100, 024005 (2019).

[31] D. J. Gross and J. H. Sloan, Nucl. Phys. B291, 41 (1987).

[32] P. Kanti, N. E. Mavromatos, J. Rizos, K. Tamvakis, and E. Winstanley, Phys. Rev. D 54, 5049 (1996).

[33] P. Pani and V. Cardoso, Phys. Rev. D 79, 084031 (2009).

[34] N. Yunes and L.C. Stein, Phys. Rev. D 83, 104002 (2011).

[35] A. Maselli, P. Pani, L. Gualtieri, and V. Ferrari, Phys. Rev. D 92, 083014 (2015).

[36] G. Papallo and H. S. Reall, Phys. Rev. D 96, 044019 (2017).

[37] G. Papallo, Phys. Rev. D 96, 124036 (2017).

[38] J. L. Ripley and F. Pretorius, Classical Quantum Gravity 36, 134001 (2019).

[39] J. L. Ripley and F. Pretorius, Phys. Rev. D 101, 044015 (2020).

[40] T. P. Sotiriou and S.-Y. Zhou, Phys. Rev. D 90, 124063 (2014).

[41] P. V. Cunha, C. A. Herdeiro, and E. Radu, Phys. Rev. Lett. 123, 011101 (2019).

[42] J. F. Delgado, C. A. Herdeiro, and E. Radu, J. High Energy Phys. 04 (2020) 180.

[43] G. Antoniou, A. Bakopoulos, and P. Kanti, Phys. Rev. Lett. 120, 131102 (2018).
[44] G. Antoniou, A. Bakopoulos, and P. Kanti, Phys. Rev. D 97, 084037 (2018).

[45] C. F. Macedo, J. Sakstein, E. Berti, L. Gualtieri, H. O. Silva, and T. P. Sotiriou, Phys. Rev. D 99 (2019).

[46] D. D. Doneva, K. V. Staykov, and S. S. Yazadjiev, Phys. Rev. D 99, 104045 (2019).

[47] H. Lu and Y. Pang, arXiv:2003.11552.

[48] B. Kleihaus, J. Kunz, and E. Radu, Phys. Rev. Lett. 106, 151104 (2011).

[49] M. Alcubierre, Introduction to 3+1 Numerical Relativity, International series of monographs on physics (Oxford Univ. Press, Oxford, 2008).

[50] K. S. Thorne, Rev. Mod. Phys. 52, 299 (1980).

[51] E. Poisson and C. M. Will, Gravity: Newtonian, PostNewtonian, Relativistic (Cambridge University Press, Cambridge, England, 2014).

[52] S. Alexander and N. Yunes, Phys. Rep. 480, 1 (2009).

[53] xActPackage, xAct: Efficient tensor computer algebra for the Wolfram Language (2002-2020).

[54] D. Brizuela, J. M. Martin-Garcia, and G. A. Mena Marugan, Gen. Relativ. Gravit. 41, 2415 (2009).

[55] F.-L. Julié and E. Berti, arXiv:2004.00003. 